\title{
Studies of Landé $\mathrm{g}_{\mathrm{J}}$-factors of singly ionized neodymium isotopes $(142,143$ and 145$)$ at relatively small magnetic fields up to $334 \mathrm{G}$ by collinear laser ion beam spectroscopy
}

\author{
Slawomir Werbowy ${ }^{1,2, a}$ and Laurentius Windholz ${ }^{2}$ \\ 1 Institut für Experimentalphysik, Technische Universität Graz, Petersgasse 16, 8010 Graz, Austria \\ 2 Institute of Experimental Physics, Faculty of Mathematics, Physics, and Informatics, University of Gdansk, \\ ul. Wita Stwosza 57, 80-308 Gdansk, Poland
}

Received 13 October 2016 / Received in final form 28 November 2016

Published online 19 January 2017

(C) The Author(s) 2017. This article is published with open access at Springerlink.com

\begin{abstract}
The Zeeman splitting at moderate magnetic fields (up to $334 \mathrm{G}$ ) of 20 lines of Nd II, covering the part $569.89 \mathrm{~nm}$ to $617.05 \mathrm{~nm}$ of the visible spectral range were investigated. We used a Doppler-free collinear laser ion beam spectroscopy technique supported by a precise computer analysis. From the Zeeman effect studies we re-investigated the Landé $\mathrm{g}_{J}$ factors for 20 odd levels between 22850 and $30002 \mathrm{~cm}^{-1}$ and 11 even levels lying between 6005 and $12460 \mathrm{~cm}^{-1}$. We compared our data with other available experimental and theoretical values. We achieved an accuracy better than that of other authors, making our data valuable for future theoretical parametric studies.
\end{abstract}

\section{Introduction}

Generally, neodymium has one of the most complex spectra of all elements with hundreds of known and classified energy levels [1]. From the studies of some infrared lines, Blaise et al. gave 687 and 684 levels for $\mathrm{Nd}$ I and Nd II [2], respectively. Few years later, Blaise and Wyart [3] have increased number of Nd II levels by 126 . Additionally, the presence of 7 stable isotopes in the naturally occurring neodymium with comparable abundances $(142-23 \%, 143-$ $13 \%, 144-25 \%, 145-9 \%, 146-18 \%, 148-6 \%$ and $150-6 \%)$ and the related significant isotope shifts makes the interpretation of highly resolved spectra even more difficult task. The isotope shifts vary from line to line and the two odd isotopes (143 and 145) with their complex hyperfine (hf) structure (both have the same large nuclear spin quantum number $I=7 / 2$ and high magnetic dipole and electric quadruple moments) contribute to the complexity.

In our opinion all fully threaten ab initio methods fails in the interpretation of the Nd I and Nd II complex spectra. Perhaps the only reasonable choice would be the Racah-Slater parametric method, where radial integrals are treated as adjustable parameters and CI parameters for two-particle electrostatic interactions at the second order of perturbation are chosen properly. In many cases this method gave theoretical energies, level designations, hyperfine constants, isotope shifts and Landé $\mathrm{g}_{J}$ factors with acceptable accuracy. Although this approach is very versatile and gives accurate results, it has one week

${ }^{a}$ e-mail: s.werbowy@ug.edu.pl point: it requires the knowledge of some spectroscopic data (usually taken from the experiment) to which the parameters could be fitted, enabling in this way the access to values of other unknown spectroscopic data. The accuracy of the experimental input data is crucial, because even small variations could lead to bad analysis of the complex atomic structure [3]. A very extensive recent theoretical interpretation of the odd parity levels in Nd II was presented by Wyart [1], who gave the designations for 596 odd levels of the mixed configurations $4 f^{3} 5 d 6 s, 4 f^{3} 5 d^{2}$, $4 f^{3} 6 s^{2}, 4 f^{4} 6 p$ and $4 f^{5}$ with $53 \mathrm{~cm}^{-1}$ mean error.

At the very beginning of studies of the atomic structure, wavelengths of observed lines and related energies of the levels together with Landé $g_{J}$ factors, determined from Zeeman effect studies, were used as the reference data in the theoretical studies. Later, together with the progress in the experimental techniques, they were complemented (or replaced) by studies of the hyperfine structure and isotope shifts, since many elements gave such opportunity. The isotope shifts and hyperfine structure constants of many Nd II levels were investigated many times in the past, also recently using high resolution Doppler free spectroscopy, e.g. [4-8].

In contrast we noticed, that there are not so many studies of the Zeeman effect of this element and the vast majority of the Landé $g_{J}$ factors were determined long time ago from Doppler limited spectroscopy with accuracy of \pm 0.01 unit [2]. This accuracy allows only the conclusion that the fine structure description is globally correct, but there are many unsatisfactory details especially in the regions where the average level spacing is small [1]. 
Due to the reasons given at the beginning of this section (the isotopic composition, large number of lines, hyperfine structure), the use of classical Doppler limited spectroscopy to study the Zeeman effect is very risky. All factors will likely affect the accuracy of the obtained data, unless one uses a specially prepared sample with one enriched isotope. Thus Doppler-effect-free methods seem to be more adequate. In our previous studies [9] we already have used a Doppler free method to study the Zeeman effect of Nd II lines. Since that time we have modified some details of the experiment in order to increase the signal to noise ratio and to observe weaker $\mathrm{Nd}$ II transitions in the wavelength region of our laser system and to study other levels. In addition, we were able to record Zeeman patterns at magnetic fields of approx. $330 \mathrm{G}$, for isotopes with mass number 143 and 145, showing hyperfine structure splitting, and for isotope 142 without hf structure.

Besides the application to the theoretical interpretation of the spectra of the element, accurate spectroscopic parameters, i.e. Landé factors, are of interest for astrophysics. Many lines were observed in stellar spectra (especially in chemically peculiar stars). In magnetic stars the presence of external magnetic fields in addition to the hyperfine structure and isotope shifts causes broadening of the lines in high resolution stellar spectra. If all the contributions to the final absorption line profile would not be properly accounted (the hyperfine structure, isotope shifts or presence of magnetic fields), this could lead to an overestimation of the abundance of the element or other important astrophysical parameters.

The aim of the present work is to re-investigate with the use of fast ion beam laser spectroscopy the Zeeman effect of some weaker lines of Nd II at weak magnetic fields using selective Doppler-free laser excitation. The Landé $g_{J}$ factors obtained in this way provide an accuracy which could be sufficient to serve as good reference data for parametric studies of Nd II.

\section{Experiment and computer analysis}

Like in previous papers $[9,10]$, the measurements were performed on the CLIBS apparatus named after its original localization, MARburg Separator MARS-II, first installed at the University of Marburg a.d. Lahn (Germany) and moved 2002 to Graz (Austria).

Details of the apparatus were given in [9-11]. A continuous-wave narrow band tunable ring-dye laser (with line width ca. $1 \mathrm{MHz}$, and typical power density $15 \mathrm{~mW} / \mathrm{mm}^{2}$ ) was stabilized and tuned to a certain transition. Neodymium ions were produced by a resistance heated oven (thermal ionization), accelerated to approx. $19 \mathrm{keV}$ energies, and an ion beam was formed and guided through a powerful electromagnetic mass separator by some ion optic components. The mass separator allowed to investigate the different isotopes separately. The laser beam was oriented anti-parallel to the ion beam. The laser light was continuously exciting fast $\mathrm{Nd}$ ions from metastable levels to high lying levels, whereupon fluorescence decay was observed through a filter to block scattered laser light.
Compared to our previous experiment $[9,10]$ we introduced several modifications (given in details in [12]), mainly connected with the improvement of parameters of the ion beam. In this way, we have increased significantly the signal-to-noise ratio $(\mathrm{S} / \mathrm{N})$ and the density of the ions in the beam. This directly allowed the investigation of previously too weak Nd II transitions with now sufficient S/N giving access to more levels to be re-investigated. The fine details of the experiment are available upon request.

A homogeneous magnetic field perpendicular to the ion beam was produced by two strong permanent neodymium magnets. The field strength and its distribution was measured with a Hall effect Gauss-meter (Applied Magnetics Laboratory model GM1A - probe model PB71-10) with an accuracy of $0.25 \%$ of the reading, and was confirmed in situ by recording the Ba II $585.368 \mathrm{~nm}\left(6 p^{2} \mathrm{P}_{3 / 2} \rightarrow 5 d\right.$ $\left.{ }^{2} \mathrm{D}_{3 / 2}\right)$ transition, for which the Landé factors of the involved levels are known with good accuracy: 1.328(8) [13] and 0.7993278(3) [14] for lower and upper level, respectively. By simply changing the alignment of the magnet bars we have produced two values of static magnetic fields, 235 (2) G and 334 (2) G, respectively, at which we have recorded the patterns.

A very important part is the proper interpretation of the experimental hf-Zeeman patterns. We have used a software developed in our group (for details see [15]), which proved its usefulness for many elements, e.g. Antimony [16,17], Lead [18], Bismuth [19-21], Lanthanum and Praseodymium $[23,24]$. In case of the $\mathrm{Nd}$ isotopes with hyperfine structure, 143 and 145, in simulations we have used the most recent hyperfine constants reported by $[5,6,8]$.

\section{Results and discussion}

In the present studies, using nearly Doppler-effect-free collinear laser-ion beam spectroscopy, we were able to analyze the Zeeman splittings of 20 lines of $\mathrm{Nd}$ II in the range between $569.892 \mathrm{~nm}$ and $617.048 \mathrm{~nm}$. Figure 1 presents typical recorded hyperfine and Zeeman structures of the observed lines. Thin lines represent the experimental traces and the thick solid lines are best fits with theoretical profiles. The typical observed line widths varied from $50 \mathrm{MHz}$ up to $120 \mathrm{MHz}$, depending on the observed transition. The main contributions to the observed line widths are coming from: natural line widths, ripple of the acceleration voltage, thermal spread of the velocity of the ions in the high temperature oven, and laser frequency instability. In case of discrete nature of data collected during experiment, the line position error can be estimated from relationship [22]: $\Delta \sigma \sim W / \sqrt{N}$, where $W$ is the half width of the line, and $N$ is the number of data points in the width $W$. Typically we used a scanning step of $5 \mathrm{MHz}$, what gives a line position accuracy between $16 \mathrm{MHz}$ and $25 \mathrm{MHz}$.

Our computer software fitting yielded accurate values of the Landé- $g_{J}$ factors of 20 odd $\mathrm{Nd}^{+}$levels between 22850 and $30002 \mathrm{~cm}^{-1}$, presented in Table 1 and 11 even levels lying between 6005 and $12460 \mathrm{~cm}^{-1}$, presented in Table 2. 



Fig. 1. Recorded Zeeman patterns of the $574.815,590.665$ and $603.127 \mathrm{~nm}$ lines of ${ }^{142} \mathrm{Nd}$ II, ${ }^{143} \mathrm{Nd}$ II, and ${ }^{145} \mathrm{Nd}$ II isotopes ( $\Delta M= \pm 1$ components at $235 \mathrm{G}$ magnetic field). The thin line represents the experimental pattern and the thick line shows the computer-generated best fit contour. The dotted line presents the calculated zero field hf pattern of the line.

The experimental uncertainties $\left(\sigma_{g}-\right.$ standard deviation) given in Tables 1 and 2 were determined from several independent measurements, obtained at different patterns of the line recorded for two polarization directions and two magnetic field values. Additionally, for certain energy levels, the presented final values were obtained from the weighted mean values of more than one line and/or isotopes, where one possible choice for the weights $w_{i}$ is: $w_{i}=\left(\sigma_{g}\right)_{i}^{-2}$. The standard error of the weighted mean was calculated as:

$$
\sigma_{\bar{g}_{w}}=\left(\sum_{i=1}^{n} w_{i}\right)^{-1 / 2}
$$

The magnetic fields were measured with certain accuracy $(\Delta B= \pm 2 \mathrm{G})$, what in principle introduces additional uncertainty to the obtained Landé factors. We estimate this contribution from the law of propagation of uncertainty as: $g_{J}(\Delta B / B)$.

The total standard error of the mean $\widetilde{\sigma}_{\bar{g}_{w}}$ includes mean standard deviations and a term which takes into account the uncertainty of determination of magnetic field, and was calculated using the relation:

$$
\widetilde{\sigma}_{\bar{g}_{w}}=\sqrt{\left(\sigma_{\bar{g}}\right)^{2}+\left(g_{J} \frac{\Delta B}{B}\right)^{2}} .
$$

Because a single level can be involved in several transitions, for two odd and six even levels Landé factors were measured at other transitions in our previous paper [9]. Although we have increased the sensitivity and studied systematically weaker $\mathrm{Nd}$ II transitions than in previous studies, we did not avoid the repetition of the measurements in case of such levels. All results are in agreement with the earlier data and can now serve as a control values. In summary, we introduce re-determined data of Landé $g_{J}$ factors for 23 more levels of Nd II.

\section{Conclusions}

According to the NIST spectral line compilation [25], in the narrow wavelength range between $569.892 \mathrm{~nm}$ and $617.048 \mathrm{~nm}$ there are 50 identified lines of Nd I and Nd II. More recent experimental studies [2] give 136 lines of $\mathrm{Nd}$ I-II (many were still unclassified) in the same wavelength range.

From parametric studies performed by Wyart [1] we know that there are approx. 260 odd levels in the 22850 $30002 \mathrm{~cm}^{-1}$ energy range. Together with our recent work [9] we have re-investigated 30 odd levels $(11.5 \%$ of all possible levels in this energy range). Globally, there are 241 even known levels of Nd II [26], besides them 55 with energies below $12460 \mathrm{~cm}^{-1}$. Present data on Landé factors together with data given in [9] gives 12 even levels ( $22 \%$ of all known levels in this range).

We have noticed discrepancies between configuration designations of four odd levels, presented in Table 3. The table contains the percentage of contribution from the leading configuration in the configuration interaction parametric studies from references $[1,3]$. However, calculated in both works Landé $\mathrm{g}_{J}$ factors are favoring the data and leading configuration assignment of [3] (please compare the data given in Tab. 1 with present experimental results). This example demonstrates that, even in general 


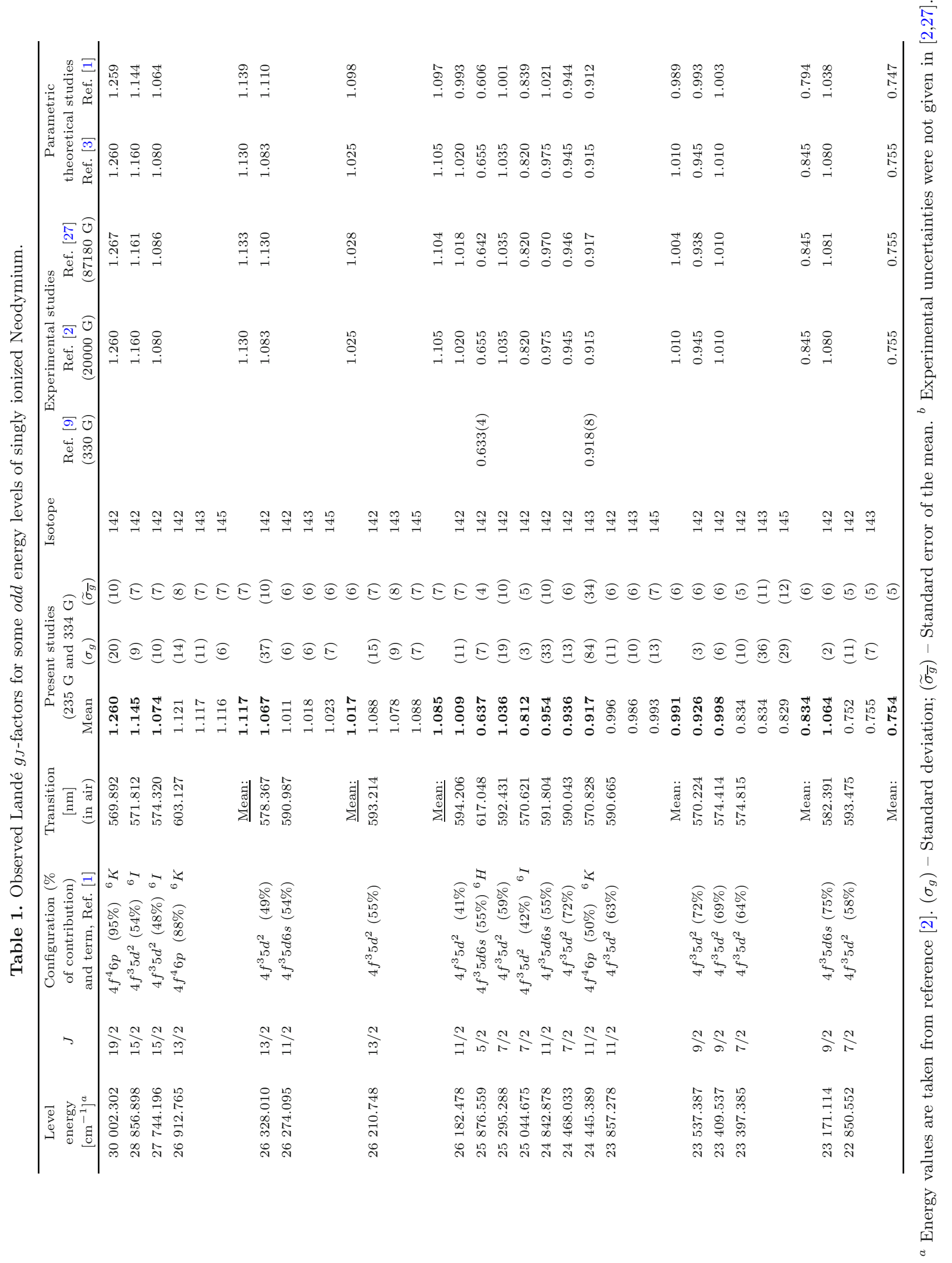




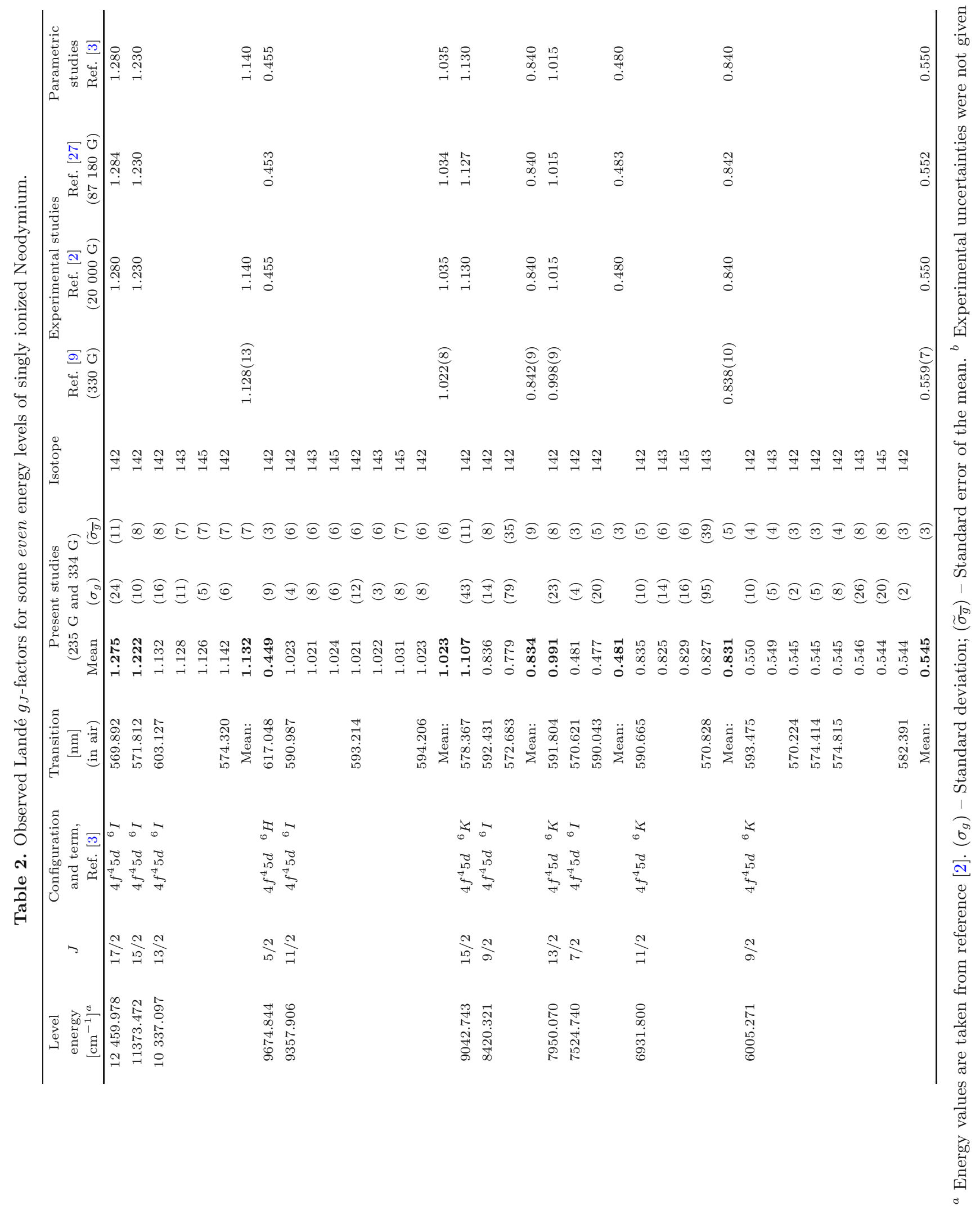


Table 3. Discrepancies of some present Nd II level designations, between two available theoretical parametric studies (Refs. [1,3]).

\begin{tabular}{|c|c|c|}
\hline $\begin{array}{l}\text { Level } \\
\text { energy } \\
{\left[\mathrm{cm}^{-1}\right]}\end{array}$ & $\begin{array}{c}\text { Configuration } \\
\text { (leading component) } \\
\text { Ref. }[3]\end{array}$ & $\begin{array}{c}\text { Configuration } \\
\text { (\% of contribution) } \\
\text { Ref. }[1]\end{array}$ \\
\hline 28856.898 & $4 f^{4} 6 p$ & $\begin{array}{ll}4 f^{3} 5 d^{2} & (54 \%) \\
4 f^{4} 6 p & (24 \%) \\
\end{array}$ \\
\hline 26328.010 & $4 f^{3} 5 d 6 s$ & $\begin{array}{ll}4 f^{3} 5 d^{2} & (49 \%) \\
4 f^{3} 5 d 6 s & (33 \%)\end{array}$ \\
\hline 25876.559 & $4 f^{4} 6 p$ & $\begin{array}{l}4 f^{3} 5 d 6 s(55 \%) \\
4 f^{4} 6 p \quad(37 \%) \\
\end{array}$ \\
\hline 25044.675 & $4 f^{4} 6 p$ & $\begin{array}{ll}4 f^{3} 5 d^{2} & (42 \%) \\
4 f^{4} 6 p & (39 \%) \\
\end{array}$ \\
\hline
\end{tabular}

the interpretation of the Nd II spectra is almost complete, there are still some inconsistencies for some levels. In the parametric studies, both authors used practically the same experimental data on Landé $\mathrm{g}_{J}$ factors, known with accuracy of \pm 0.01 unit. In case our present experimental studies we have achieved a better accuracy of the determined Landé $\mathrm{g}_{J}$ factors than other authors.

Summarizing, there is still lot of work to be done in the field of studies of the Landé $g_{J}$ factors in Nd II. However, we hope that the presented data may be sufficient and encouraging for performing more extensive theoretical parametric studies for a better interpretation of the complex Nd II spectrum.

Unfortunately, in the presented method of Dopplerfree laser induced spectroscopy, the excitation is limited by the fact that only metastable low-lying states can serve as lower states of the laser excitation. Thus only a limited number of transitions can be studied. Recently, we have demonstrated $[23,24]$ an alternative experimental method (use of a of liquid nitrogen cooled hollow cathode discharge lamp with selective optogalvanic and fluorescence laser spectroscopy) to perform similar Zeeman effect studies of the rare earth element atoms (lanthanum and praseodymium), characterized also by very dense spectra. Although this method is Doppler-limited it can give access to a much higher number of levels to be excited. However, this method (the hollow cathode source) would likely fail in case of neodymium spectra when using natural Nd due to the presence of 7 isotopes with comparable abundances and isotope shifts associated with this. Additionally, the hyperfine structure components of the two 143 and 145 isotopes makes the patterns even more blurred. The problem could be solved by (expensive) enriched Nd samples (even isotopes with $I=0$ ) for the inner wall of the hollow cathode.

The present work was supported by WissenschaftlichTechnisches Abkommen Österreich-Polen, Projects Nos. 21/ 2012 and 12/2014. The interest of Prof. Dr. H. Hühnermann, University of Marburg/Lahn, is acknowledged.

\section{References}

1. J.F. Wyart, Phys. Scr. 82, 035302 (2010)

2. J. Blaise, J. Chevillard, J. Vergi, J.F. Wyart, Spectrochim. Acta B 268, 333 (1970)

3. J. Blaise, J.F. Wyart, Phys. Scr. 29, 119 (1984)

4. L. Maosheng, M. Hongliang, C. Miaohua, L. Fuquan, T. Jiayong, Y. Fujia, Phys. Rev. A 62, 052504 (2000)

5. S.D. Rosner, D. Masterman, T.J. Scholl, R.A. Holt, Can. J. Phys. 83, 841 (2005)

6. N. Anjum, Ph.D. thesis, Graz University of Technology, Graz, 2012

7. W. Koczorowski, E. Stachowska, B. Furmann, D. Stefaéska, A. Jarosz, A. Krzykowski, Spectrochim. Acta B 60, 447 (2005)

8. N. Anjum, N. Akhtar, H. Hühnermann, L. Windholz, J. Phys. B 48, 015003 (2015)

9. S. Werbowy, H. Hühnermann, J. Kwela, L. Windholz, J. Quant. Spec. Rad. Trans. 166, 102 (2015)

10. S. Werbowy, N. Anjum, H. Hühnermann, J. Kwela, L. Windholz, Phys. Rev. A 90, 032515 (2014)

11. N. Akhtar N, N. Anjum, H. Hühnermann, L. Windholz, Eur. Phys. J. D 66, 264 (2012)

12. S. Werbowy, L. Windholz, J. Quant. Spec. Rad. Trans. 187, 267 (2017)

13. O. Poulsen, P.S. Ramanujam, Phys. Rev. A 14, 1463 (1976)

14. K.H. Knöll, G. Marx, K. Hübner, F. Schweikert, S. Stahl, C. Weber, G. Werth, Phys. Rev. A 54, 1199 (1996)

15. S. Werbowy, J. Kwela, R. Drozdowski, J. Heldt, Eur. Phys. J. D 39, 5 (2006)

16. S. Werbowy, J. Kwela, J. Phys. B 43, 065002 (2010)

17. Ł.M. Sobolewski, S. Bouazza, J. Kwela, Eur. Phys. J. D 70, 20 (2016)

18. S. Werbowy, J. Kwela, J. Phys. B 42, 065002 (2009)

19. S. Werbowy, J. Kwela, Eur. Phys. J.: Special Topics 144, 179 (2007)

20. Ł.M. Sobolewski, S. Werbowy, J. Kwela, J. Opt. Soc. Am. B 31, 3038 (2014)

21. S. Werbowy, J. Kwela, Phys. Rev. A 77, 023410 (2008)

22. S.P. Davis, M.C. Abrams, J.W. Brault, Fourier Transforme Spectrometry (Academic Press, 2001)

23. S. Werbowy, C. Güney, L. Windholz, Spectrochim. Acta B 116, 16 (2016)

24. S. Werbowy, C. Güney, L. Windholz, J. Quant. Spectrosc. Rad. Trans. 179, 33 (2016)

25. W.F. Meggers, C.H. Corliss, B.F. Scribner, Natl. Bur. Stand. Monograph, Nat. Bur. Stand., US 145, 600 (1975)

26. W.C. Martin, R. Zalubas, L. Hagan, Nat. Stand. Ref. Data Ser. 60, 422 (1978) (Nat. Bur. Stand., U.S., 1978)

27. W.E. Albertson, G.R. Harrison, J.R. McNally Jr., Phys. Rev. 61, 167 (1942)

Open Access This is an open access article distributed under the terms of the Creative Commons Attribution License (http://creativecommons.org/licenses/by/4.0), which permits unrestricted use, distribution, and reproduction in any medium, provided the original work is properly cited. 